INTERNATIONAL BULLETIN OF BACTERIOLOGICAL NOMENCLATURE AND TAXONOMY

Vol. 15, No. 2

April 15, 1965

pp. $97-106$

\title{
SUR LA NOMENCLATURE ET LA TAXINOMIE DU BACILLE DE MALASSEZ ET VIGNAL
}

H. H. Mollaret

\section{Institut Pasteur, Paris}

ABSTRACT. The designation "pseudotubercular" has been incorrectly applied to the macroscopic lesions produced by many species of bacteria. Further, the frequent inclusion of "pseudotuberculosis" as a specific epithet inappropriately in names of bacteria has led to much confusion. This is particularlytrue in the many names applied to the organism described by Malassez and Vignal (1883). Reasons for recognition of the following names of genera and species segregated from the genus Pasteurella are detailed.

Yersinia Van Loghem 1944

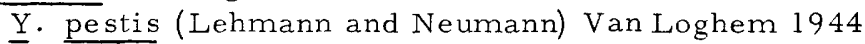

(Yersin's organism of plague);

$\underline{Y}$. rodentium Van Loghem 1944 (the bacillus of

Malassez and Vignal);

$\underline{Y}$. enterocolitica (Schleifstein and Clark) Frederiksen.

Francisella Dorofeev 1947

F. tularensis (McCoy and Chapin) Dorofeev.

La multiplicité des noms proposés pour désigner le germe découvert en 1883 par Malassez et Vignal rend bien compte des hésitations successives des auteurs sur la position à lui attribuer dans la Systématique: bacille de la tuberculose zoogléique (Malassez et Vignal 1883), Strepto-bacille (Dor 1888), Bacillus pseudotuberculosis (Pfeiffer 1889; Eisenberg 1891), Streptobacillus pseudotuberculosis rodentium (Preisz 1894), Bacterium pseudotuberculosis rodentium (Lehmann et Neumann 1896), Bacterium pseudotuberculosis (Migula 1900), Bacillus pestis intestinalis caviae (Byloff 1906), Bacillus pseudopestis (Pribram et Plasaj 1921), Corynebacterium rodentium (Bergey et coll. 1923), Corynebacterium pseudotuberculosis (Bergey et coll. 1925), Corynebacterium 
pseudotuberculosis rodentium (Kelser 1933), Malleomyces pseudotuberculosis rodentium (Pribram 1933), Pasteurella pseudotuberculosis (Topley et Wilson 1936), Yersinia rodentium (Van Loghem 1944-1945), Cillopasteurella pseudotuberculosis rodentium (Prévot 1948).

En outre, selon Haupt $(1934,1935)$ les appellations suivantes: Bacterium pfaffi (Hadley 1918), Eberthella pfaffi (Bergey et coll. 1925) et Shigella pfaffi (Weldin 1927; Bergey et coll. 1930) "sont des synonymes probables du Bacterium pseudotuberculosis des rongeurs de Pfeiffer."

La dénomination Shigella pseudotuberculosis proposée par Haupt (1935)* a été adoptée par I'Ecole veterinaire de Giessen (Haupt et Roots 1954; H. Muller 1954; W. Bossaller 1955; A. Weis 1955) et se retrouve dans nombre de traités: Cohrs, Jaffe et Meessen (1958); Nieberle et Cohrs (1961); Wetzel et Rieck (1962).

Cette diversité d'appellations a créé une confusion qu'aggravent encore les synonymies malheureuses qui se rencontrent à propos du bacille de Preisz-Nocard [Corynebacterium pseudotuberculosis (Nocard 1885), Pseudotuberculose-Bakterie (Preisz 1891), Bacillus pseudotuberculosis ovis (Lehmann et Neumann 1896), Corynebacterium pseudotube rculosis ovis (Hauduroy 1937) ], du bacille de Kutscher [Bacillus pseudotuberculosis murium (Kutscher 1894; Lehmann et Neumann 1896); Corynethrix pseudotuberculosis murium (Bongert 1901)] et des germes isolés par Courmont (Bacillus pseudotuberculosis similis Kruse 1896) ou par Du Cazal et Vaillard (Bacillus pseudotuberculosis liquefaciens Kruse 1896).

La confusion se trouve portée à son comble par l emploi de l'epithète "pseudotuberculeux." Ce terme malencontreux qui ne désigne que des lésions macroscopiques non spécifiques a été et demeure utilisé indifféremment à propos des agents étiologiques les plus variés, inertes ou vivants, parasitaires, mycosiques ou bactériens et, parmi ces derniers, concerne aussi bien le bacille de Malassez et Vignal que celui de Preisz-Nocard ou que le bacille de Whitmore, le bacille pyocyanique, le bacille de la morve, Pasteurella tularensis, Pasteurella pestis, Proteus morganii, certaines Salmonellae etc.

$\because$ Recemment (1964) Haupt a abandonné cette dénomination au profit de Cillopasteurella pseudotuberculosis. 
Cette appellation, condamnée dès 1899 par le Subcommittee of the Pathological Society of London appointed to consider the nomenclature of the conditions sometimes described as pseudotuberculosis, n'en a pas moins continué d'être employée malgré les dénonciations périodiques de son impropriété. Reimann en 1932, puis Meyer en 1958, pour tenter de limiter les confusions, ont proposé d'en réserver l'emploi au seul bacille de Malassez et Vignal, mais ces proposition sont restées lettre morte.

Ce problème de nomenclature se double d'un problème de systématique: tout à tour rangé dans les genres Bacillus, Bacterium, Corynebacterium, Malleomyces, Eberthella Shigella, le bacille de Malassez et Vignal occupe actuellement dans le genre Pasteurella une position que les connaissances actuelles ne permettent plus de lui conserver. Berlin et Borzenkov (1938), Thal (1954), Talbot et Sneath (1960), entre autres, ont souligné à juste titre les incorrections de la Nomenclature et de la Taxinomie ausi bien à l'endroit du bacille de Malassez et Vignal qu'à celui du genre Pasteurella en général; Steel (1963) a également signalé la fréquence des confusions entre $\underline{P}$. pseudotuberculosis et $\underline{P}$. septica. Nous insistons à notre tour sur la double necessité de rejeter l'epithète "pseudotuberculeux" et de retirer du genre Pasteurella le bacille de Malassez et Vignal et le bacille de Yersin.

L'examen du tableau ci-joint montre suffisamment qu'il n'existe, entre les bacilles de Yersin et de Malassez et Vignal d'une part et Pasteurella septica ou multocidad'autre part, aucun caractère justifiant que ces trois germes demeurent plus longtemps réunis dans le genre Pasteurella; Girard (1942) l'avait déjà souligné: "le développement des bacilles pesteux et pseudotuberculeux en eau de levure et en eau de pomme de terre, l'absence de floculation du mélange sérum antipesteux-filt rat de culture de Pasteurella, l'absence totale de lyse des Pasteurella par un bactériophage antipesteux dont la polyvalence et l'activité sont très marquées, ajoutent encore au faisceau d'arguments invoqués pour séparer des Pasteurella le bacille de Yersin et celui de Malassez et Vignal. Que leur reste-t-il. en effet, de commun? La coloration bipolaire, au nom de laquelle ils ont été jadis intégrés dans le même genre. Reconnaissons que ce caractère n'a aujourd'hui rien de spécifique. " 
Ajoutons que $\underline{P}$. septica produit de l'indole, fermente le saccharose, respecte le glycerol, le rhamnose et l'esculine, possède une tétrathionate-réductase, une fumarate-réductase (Pichinoty, Le Minor et Mollaret) et reste dépourvue de $\beta$-galactosidase (Mollaret et Le Minor).

La composition en bases nucléiques de l'acide desoxyribonucléique, exprimée en guanine + cytosine (G. C. \%) sépare nettement $\underline{P}$. septica $(34-36 \%)$ de $\underline{P}$. pestis $(46-48 \%)$ (Marmur, Falkow et Mandel).

Enfin, l'absence de rapports antigéniques et la différence de comportement chez les insectes hématophages confirment encore l'opposition entre $\underline{P}$. septica d'une part et les bacilles de Malassez et Vignal d'autre part.

A l'inverse, comme nous l'avons déjà développé (1962), tout concourt à rapprocher le bacille de Yersin du bacille de Malassez et Vignal: analogies morphologiques, culturales, biochimiques et antigéniques, sensibilité aux mêmes antibiotiques et surtout aux mêmes bactériophages, analogie de comportement chez Xenopsylla cheopis, analogie physiopathologique enfin avec la parenté de leurs toxines et le parallélisme du leurs lésions histologiques. La seule différence n'est donc en définitive qu'une question de degré dans le pouvoir pathogène, encore ce critère est-il restreint par l'existence de souches de bacille de Yersin peu pathogènes ou de souches perdant partie ou même tout de leur pouvoir pathogène après contact avec le bactèriophage.

D'une facon générale, la plupart des caractères classiques de differenciation de l'un ou de l'autre germe peuvent être modifiés sous l'action du bactériophage; ainsi Korobkova (1937) a pu faire acquérir au bacille de Yersin 1'aptitude à fermenter le rhamnose et Brunet (1952) a pu faire perdre au bacille de Malassez et Vignal son appareil locomoteur. Nous n'avions pendant longtemps retenu en définitive que deux critères de différenciation: l'aptitude à décomposer l'urée et la mobilité, apanages toutes deux du bacille de Malassez et Vignal. Mais la portée théorique de cette mobilité est singulièrement affaiblie en raison de sa disparition dans certaines conditions d'expériences comme de son étroite subordination aux conditions de température; il en est de même avec le bacille de Yersin dont nombre de caractères et les exigences nutritives en particulier sont fonction de la température. En ce qui concerne l'uréase dont le bacille de Malassez et Vignal avait longtemps paru être, des deux 
Table I. Principaux caractères de différenciation de Pasteurella septica et des Yersinia.

\begin{tabular}{|c|c|c|c|c|}
\hline & $\begin{array}{l}\text { Yersinia } \\
\text { malassezii } *\end{array}$ & $\begin{array}{l}\text { Yersinia } \\
\text { enterocolitica }\end{array}$ & $\frac{\text { Yersinia }}{\text { pestis }}$ & $\frac{\text { Pasteurella }}{\text { septica }}$ \\
\hline Mobilité à $20^{\circ}$ & + & + & 0 & 0 \\
\hline Uréase & + & + & $\mathrm{d}$ & 0 \\
\hline Indole & 0 & 0 & 0 & + \\
\hline Pomme de terre & + & + & 0 & 0 \\
\hline Eau de levure & + & + & + & 0 \\
\hline Saccharose & 0 & + & 0 & + \\
\hline Rhamnose & + & 0 & $\mathrm{~d}$ & 0 \\
\hline Glycérine & + & + & $\mathrm{d}$ & 0 \\
\hline Esculine & + & 0 & + & 0 \\
\hline Rouge de méthyle & + & + & + & 0 \\
\hline Tetrathionate réductase & 0 & 0 & 0 & + \\
\hline$\beta$-galactosidase & + & + & + & 0 \\
\hline Rapports antigéniques & + & 0 & + & 0 \\
\hline Rapports bactériophagiques & + & 0 & + & 0 \\
\hline Persistance chez Xenopsylla cheopis & + & $?$ & + & 0 \\
\hline
\end{tabular}

* Yersinia rodentium 
germes, le seul pourvu, nous connaissons maintenant des souches de peste douées de la même propriété (Mollaret 1964).

Nous estimons donc que le bacille de Yersin et le bacille de Malassez et Vignal, actuellement séparés et classés, l'un dans le genre Cillopasteurella (Prevot 1948), l'autre dans le genre Pasteurella(Trevisan 1887), doivent être rapprochés, comme l'avait déjà réclamé Tumanskij en 1939 et réunis dans le genre Yersinia proposé par Van Loghem (1944-45, 1946). Cet auteur a suggéré les dénominations respectives de Yersinia pestis et de Yersinia rodentium; à cette dernière, qui implique une origine animale trop limitée, nous préférons celle de Yersinia malassezii et nous proposons cette modification conformément au principe 1 du Code Inte rnational de Nomenclature Bactérienne selon lequel "doit être évité ou rejeté l'usage de termes et de noms qui peuvent être les causes d'erreurs ou d'ambiguité et être source de confusion," ce qui est très exactement le cas de toutes les appellations proposées jusqu'à ce jour.

Dans ce genre Yersinia doit prendre également place sous la dénomination de Yersinia enterocolitica (Frederiksen 1964) l'espèce antérieurement décrite sous les appellations suivantes: Bacterium enterocoliticum (Schleifstein et Clark 1947), Pasteurella pseudotuberculosis Type "b" (Dickinson et Mocquot 1961), Pasteurella "X" (Daniels et Goudzwaard 1963), Germe "X" (Mollaret et Chevalier 1964).

Dans ce démembrement du genre Pasteurella l'adoption du genre Yersinia doit s'accompagner de celle du genre Francisella (Dorofeev 1947; Olsufiev et coll. 1959) pour y ranger l'agent de la tula rémie, Pasteurella tularensis en l'état actuel de la Nomenclature et qui, pas plus que les bacilles de Yersin et de Malassez et Vignal, n'est à sa juste place dans le genre Pasteurella.

\section{BIBLIOGRAPHIE}

Bergey et coll. Bergey's Manual of Determinative Bacteriology. Williams and Wilkins Co., Baltimore. Ière éd. 1923; 2è ed. 1925; 3è ed. 1930.

Berlin, A. L. and A.K. Borzenkov, 1938. Caractères fermentaires des souches mongoles de B. pestis. Rev. microbiol. (Saratov), XVII, $\mathrm{n}^{\circ} 3-4,215-225$. 
Bongert, R. 1901. Corynethrix pseudotuberculosis murium, ein neuer pathogener Bacillus für Mäuse. Beitrag zur pseudotuberculose der Nagethiere, Z. Hyg. Infekt. Kr., 37: $449-475$.

Bossaler, W. 1955. Untersuchungen über die antigene Struktur der Shigella pseudotuberculosis (syn. Pasteurella pseudotuberculosis), thèse vétérinaire, Giessen.

Brunet, J. 1952. Relation entre les variantes de Pasteurella pestis et Pasteurella pseudotuberculosis d'une part, et le bacteriophage d'autre part, thèse médecine, Paris.

Byloff, K. 1906. Über eine pestähnliche Erkrankung der Meerschweinchen, Zbl. Bakt., I, Abt. Orig. 41:707-712 et $42: 5-13$.

Code International de Nomenclature Bacterienne. 1948. Ann. Inst. Pasteur, 74, $\mathrm{n}^{\circ} 4,271-299$.

Cohrs, P., R. Jaffe, and M. Meessen. 1958. Pathologie der Laboratoriumstiere, Springer édit., Berlin.

Courmont, J. 1889. Sur une tuberculose microbienne et particulière du boeuf, C. R. Soc. Biol. (Paris), I, 9e série, 215-218.

Daniëls, J.J.H. M. and C. Goudzwaad. 1963. Enkele Stammen van een op Pasteurella pseudotuberculosis gelijkend niet geidentificeerd species, geisoleerd bij knaadieren. T. Diergeneesk., 88, $\mathrm{n}^{\circ} 2,96-102$.

Dickinson, A. B. and G. Mocquot. 1961. Studies on the bacterial flora of the alimentary tract of pigs. I: Enterobacteriaceae and other Gram negative bacteria. Jour. Appl. Bact. 24(3):252-284.

Dor, L. 1888. De la tuberculose streptobacillaire du lapin et du cobaye. C. R. Soc. Biol. (Paris), V, 8e série, 449-451.

Dorofeev, K. A. 1947. On classification of tularemic bacteriae. Symp. Res. Works of Inst. Epidem. Mikrobiol., (Chita), 1:177-178.

Du Cazal and Vaillard. 1891. Sur une maladie parasitaire de l'homme transmissible au lapin. Ann. Inst. Pasteur, $\underline{V}, n^{\circ} 6,353-361$.

Eisenberg, J. 1891. Bakteriologische Diagnostik. L. Voss édit., Hambourg and Leipzig.

Frederiksen, W. 1964. A study of some Yersinia pseudotuberculosis-like bacteria ("Bacterium enterocoliticum" and "Pasteurella X") Proc. XIV Scandinavian Congr. 
Pathol. and Microbiol., Oslo 1964, Universitetsforlaget Trykningssentral, Oslo.

Girard, G. 1942. Sur quelques nouveaux caractères differenciant les bacilles de la peste et de la pseudotuberculose des Pasteurella. Ann. Inst. Pasteur 68(9-10):476-478.

Hadley, P.M., M. W. Elkins, and D.W. Caldwell. 1918. The colon typhoid intermediates as causative agents of diseases in birds. I: The paratyphoid bacteria. Agr. Expt. Sta. Rhode Island State Coll. Bull. $N^{\circ} 174,216$. Hauduroy, P. 1937. Dictionnaire des Bactéries pathogenès. lère ed., Masson édit., Paris.

Haupt, H. 1934. Bacterium pfaffi Hadley 1918, Bacillus pseudotuberculosis Eisenberg 1891. Zbl. Bakt. I. Abt. Orig. , 132:349.

1935. Zur Systematik der Bakterien. Ergbn.

Hyg. Bakt., 17:175-230.

- 1964. Medizinisch-bakte riologische Diagnostik.

F. Enke édit., Stuttgart.

and E. Roots. 1954. Klassification der Bakterien und Viren, in: Tafeln des Veterinär-hygienischen und Tierseucheninstitutes der Justus Liebig-Hochschule in Giessen. (ronéo).

Kelser, R. 1938. Manual of Veterinary Bacteriology. Baillière Tindall and Cox, édit., Londres.

Korobkova, S.I. 1937. Action du bactériophage pesteux sur les variétés du b. pesteux et obtention de souches avirulentes. Rev. Microbiol. Saratov, XVI, $N^{\circ}$ 1-2, 3-17.

Kruse, W. 1896. Bacillen, in Flügge: Die Mikroorganismen. F.C.W. Vogel édit., Leipzig, t. II, 454-455.

Kutscher. 1894. Ein Beitrag zur Kenntnis der bacillären Pseudotuberkulose der Nagetiere. Z. Hyg. Infekt. Kr. 18:327-341.

Lehmann, K. B. and R. O. Neumann. 1896. Atlas und Grundriss der Bakteriologie und Lehrbuch der speziellen bacteriologischen Diagnostik. Lehmanns édit., Munich.

Malassez and Vignal. 1883. Tuberculose zoogleique. Arch. Phys. Norm. et path., 2, 2e semestre, 3e série, 369411 .

Marmur, J., S. Falkow and M. Mandel. 1963. New approaches to bacterial taxonomy. Ann. Rev. Microbiol. $17: 329-372$.

Meyer, K.F. 1958. In: Dubos, R.J., Bacterial and Mycotic Infection of Man. Lippincott édit., Philadelphia. 
Migula, W. 1900. System der Bakterien. G. Fischer édit., Jena.

Mollaret, H. H. 1962. Le Bacille de Malassez et Vignal. Caractères culturaux et biochimiques, thè se médecine, Paris.

, A. Chevalier, and M. C. Deplanche. 1964. Contribution à l'étude d'un nouveau groupe de germes proches du bacille de Malassez et Vignal. I: Caractères culturaux et biochimiques. Ann. Inst. Pasteur, 107(1):121-127. and L. LeMinor. 1962. Recherche $\overline{\text { de }}$ la $\beta$-galactosidase chez les différentes Pasteurella et conséquences quant à leur taxinomie. Ann. Inst. Pasteur 102(5):649-52. , Nguyen Van Ba, M. Vandekerkove, Y. Karimi, et M. Eftekhari. 1964. Sur l'uréase du bacille de Yersin. Ann. Inst. Pasteur 107(3):424-429.

Muller, H. 1954. Untersuchungen über das Vorkommen von Toxinen bei der Shigella pseudotuberculosis (syn. Pasteurella pseudotuberculosis) thèse vétérinaire. Giessen.

Nieberle, K. and P. Cohrs. 1961. Lehrbuch der speziellen pathologischen Anatomie der Haustiere. G. Fisher édit., Jena.

Olsuf'ev and coll. 1959. Comparative studies of strains of B. tularense in the Old and New World and their taxonomy. Jour. Hyg. Epidem. (Praha) 3:138-149.

Pfeiffer, A. 1889. Ueber die bacilläre Pseudotuberkulose bei Nagethieren. G. Thieme édit., Leipzig.

Philip, C. B. and C. R. Owen. 1961. Comments on the nomenclature of the causative agent of tularemia. Int. Bull. Bact. Nomen. Tax. 11:67-72.

Pichinoty, F., L. Le Minor, and H. H. Mollaret. Recherche de la fumarate réductase chez les bactéries anaérobies facultatives. Canad. Jour. Microbiol., à paraitre.

Preisz, H. 1891. Jour. Med. Vet. 16:563.

- 1894. Recherches comparatives sur les pseudotuberculoses bacillaires et une nouvelle espèce de pseudotuberculose. Ann. Inst. Pasteur, $\underline{8}(4): 231-255$.

Prévot, A.R. 1948. Manuel de classification et de détermination des bactéries anaérobies. (Monographie de l'Institut Pasteur), Masson édit., 2e ed. Paris. 
Pribram, E. 1933. Klas sifikation der Schizomyceten. Leipzig und Vienne. und S. Plasaj. 1921. Zur Systematik der Bakterien der hämor rhagischen Septikämie. Zbl. Bakt. I. Abt. Orig. 85, Beiheft, 117-121.

Reimann, H. A. 1932. Further studies on B. pseudotuberculosis. Amer. Jour. Hyg. 16:206-214.

Schleifstein, J. and M.E. Clark. 1947. Interesting observation (Bacterium enterocoliticum). A. R. Div. Lab. Res. N. Y. St. Dept. Hlth., 71 .

Steel, K.J. 1963. Serological classification of Pasteurella pseudotuberculosis and Pasteurella septica. Mth. Bull. Minist. Hlth. Lab. Serv. 42:176-179.

Sub-Committee of the Pathological Society of London appointed to consider the nomenclature of the conditions sometimes described as pseudotuberculosis. Trans. path. Soc. Lond. 1899, 50:331-360 et 361-364.

Talbot, J.M. and P.H. A. Sneath. 1960. A taxonomic study of Pasteurella septica, especially of strains isolated from human sources. Jour. Gen. Microbiol. 22:303-311.

Thal, E. 1954. Untersuchungen über Pasteurella pseudotuberculosis, unter besonderer Berücksichtigung ihres immunologischen Verhaltens. thèse veter. Berlingska Bokt ryckeriet, Lund.

Topley and Wilson. 1936. The Principles of Bacteriology and Immunity. E. Arnold édit., $2 \mathrm{e}$ ed., Londres.

Trevisan, V. 1889. Generi e le specie delle Batteriacee. Milan.

Tumanskij, V. 1939. Contribution à l'étude de la classification du B. pseudotuberculosis rod. Pfeiffer, Rev. Microbiol. (Saratov), 18(3-4):236-238.

Van Loghen, J.J. 1944-45. The classification of plague bacillus. Antonie v. Leeuwenhoek 10:15-16. - 1946. La classification du bacille pesteux. Ann. Inst. Pasteur 72(11-12:975.

Weis, A. 1955. Immunisie rungsversuche mit Shigella pseudotuberculosis, (syn. Pasteurella pseudotuberculosis), thè se vétérinaire. Giessen.

Weldin. 1927. cité par Haupt, 1935.

Wetzel, R. et W. Rieck. 1962. Krankheiten des Wildes. Paul Parey édit., Hambourg. 